\title{
A SIMULATION-OPTIMIZATION APPROACH FOR ASSESSING THE IMPACT OF A NEW CLIENT ON THE 3PL'S LAST-MILE DELIVERY PERFORMANCE
}

\author{
Miloš Ružić ${ }^{1}$, Dražen Popović ${ }^{\text {, }}$ Milorad Vidović ${ }^{3}$, Dušan Bešić ${ }^{4}$ \\ ${ }^{1,2,3}$ University of Belgrade, Faculty of Transport and Traffic Engineering, Vojvode Stepe 305, 11000 \\ Belgrade, Serbia \\ ${ }^{4}$ Milšsed Group, Bulevar Zorana Djindjića 121, Belgrade, Serbia
}

Received 30 November 2020; accepted 20 January 2021

\begin{abstract}
An increasing number of companies are turning their attention to the company's development through various analytics, and logistics companies are not an exception. It is through quality analysis that companies can gain a market advantage over their competitors. This paper focuses on analyzing the distribution system of one of the largest logistics companies in Serbia. The analysis refers to the current company state, after which was presented the idea of defining the price that the company can offer to its future customers. Pricing is done using a simulation-optimization model developed in Python 3.7. In addition to the simulation itself, great importance is given to the development of a heuristic model for solving vehicle routing problems that contain a combination of the nearest neighbour algorithm with 2-OPT, Swap, Relocate, and Path Relocate route enhancement algorithms.
\end{abstract}

Keywords: simulation, heuristics, optimization, VRP.

\section{Introduction}

One of the problems that logistics companies face daily is the distribution of goods. Distribution aims to deliver the goods to end users with minimal cost but the maximum level of quality of service (Mičeta et al., 2019). In addition to the fact that the last mile in the supply chain requires the highest financial costs, it is also the least efficient and most polluted part of the supply chain (Gevaers et al., 2014), which further indicates the importance of optimizing it. A company that wants to reach a competitive price in the market while earning some profit must continuously improve its distribution process. Another problem that companies are facing is the pricing of the service they offer to their customers. On the one hand, it is necessary to provide a sufficiently low price that is competitive in the market. At the same time, on the other, it has to be sufficiently high to be profitable for the logistics provider. This is where it is necessary to find a balance and enter the market with a fair offer.

For a company to determine the price of the service mentioned above, it is necessary to be informed about the current state of distribution in the company. After that, the company has to guess how the eventual arrival of a new client would affect her. One of the beneficial methods for solving such

${ }^{2}$ Corresponding author: d.popovic@sf.bg.ac.rs 
problems can be a simulation. It is one of the most powerful methods whose use provides quality analysis as well as quantification and sizing of logistics systems in general (Drenovac et al., 2020). In order to present the simulated system as close to real as possible, it is possible to use data from the past to form the simulation. With adequate processing and analysis, this data can significantly contribute to the company in its decision-making process, so companies store them and keep them in different databases. For this paper, data on deliveries over a period of one month from one logistics were provided from one company in Serbia. Data can be used to create an image of the current state of distribution but also to simulate the arrival of a new client. In addition to simulating a new customer's entry into the distribution system, much attention is given to the Vehicle Routing Problem (VRP). In practice, a large number of mathematical and heuristic models can solve this problem (Teodorović, 2016). Although mathematical models provide optimal solutions, they are not often used in practice because they belong to the group of NP-hard models. On the other hand, there are plenty of heuristics that can provide a sufficiently good solution for a short period, which is close to optimal and is more often used in practice.

The paper aims to propose one approach that can help a company define service cost to new clients. The idea is first to establish the current state of distribution based on past data and then use the simulation to define the appearance of the system after the arrival of a new client. Comparative analysis of the system before and after the client's arrival shows the impact of his arrival on the distribution costs. It should also be mentioned that the model itself was developed using Python 3.7 programming language in the Spyder environment. The paper is organized into two segments. The first part deals with the formation of a heuristic model for solving VRP, as well as the definition of a simulation model. In the second part of the paper, after explaining the problem and analyzing the input data, the use of the VRP model is presented, and thus the current state of the company is presented. After that, the simulation model's input parameters were defined, after which again the condition was obtained, using the VRP model, which depicts the system upon arrival of a new user.

\section{Problem Description}

First of all, it is necessary to define the problem itself and to show the input data related to this example. As mentioned above, the case refers to the distribution of goods by a logistics company that has a regional centre where the model was tested. Products are distributed from this centre to a large number of objects grouped into mini-clusters by ZIP code (later referred to as PTT). It is these PTT clusters that represent vehicle routing locations, and vehicle velocity and distance between all objects within the PTT location are approximate values. In the observed case, average travel speed and distance between two objects in one PTT cluster is $22 \mathrm{~km} / \mathrm{h}$ and $2.5 \mathrm{~km}$ respectively (e.g. for 20 objects in one PTT cluster vehicle must travel $19^{*} 2.5 \mathrm{~km}=47.5 \mathrm{~km}$ in total, where travel time is approximately 130 minutes). For each PTT site, coordinates are given by which a matrix was obtained using the Euclidean distance. Data on all deliveries made within one month are provided, which, in addition to the delivery date, contains information on the delivery number, facility, PTT location, weight, and number of pallets in delivery. The distribution is realized with nine different 
types of vehicles whose characteristics are shown (Table 1). It should be mentioned that the company strategy is that all deliveries over $2430 \mathrm{~kg}$ and eight pallets are delivered directly, and other distributions that are less than the specified limit are routed. In addition to the payload limit and the number of vehicle pallets, a driver's working time limit is 9 hours. Working hours are calculated based on the distance travelled by the vehicle and the length of stay on the premises. The average speed of a vehicle between PTT locations is $65 \mathrm{~km} / \mathrm{h}$, while the dwell time of a vehicle in a PTT location depends on the number of objects the vehicle needs to serve within the PTT. The time required to service one facility within a PTT site is also an approximate value and is $10 \mathrm{~min}$. After the defined input data, the VRP model mentioned above should be applied in order to obtain, first of all, the current state of distribution. It relates to the case before the arrival of a new client in the system, and this is done in the next part of the paper.

Table 1

Vehicles used for Distribution

\begin{tabular}{|l|c|c|c|c|c|c|c|c|c|}
\hline Vehicle & V1 & V2 & V3 & V4 & V5 & V6 & V7 & V8 & V9 \\
\hline Capacity [kg] & 729 & 1166 & 1458 & 2430 & 3402 & 4860 & 6804 & 9720 & 23328 \\
\hline Num. of Pallets & 1 & 4 & 5 & 8 & 12 & 15 & 17 & 20 & 33 \\
\hline Costs $[\mathbf{m} . \mathbf{u} . / \mathbf{k m}]$ & 0.189 & 0.212 & 0.244 & 0.284 & 0.316 & 0.341 & 0.362 & 0.378 & 0.394 \\
\hline
\end{tabular}

\section{Simulation-Optimization Model}

The most significant contribution to this work is the development of a simulationoptimization model aimed at defining the optimal price of the service offered to clients. The model itself consists of two parts. First of all, a heuristic VRP model related to vehicle routing has been developed, and its application gives the current state of distribution in the company. Due to the complexity of observed study and the necessity for short computational time (in reality, company should obtain the results regarding the impact of new client shortly after that client request for service price) we have developed fast computational heuristic approach that relies on fast and proven algorithms (where these algorithms produce sufficiently good solutions). The second part of the model refers to the concept of simulating the arrival of a new user to the system. In the following, both heuristic and simulation approaches are presented.

\subsection{Heuristic VRP Model}

VRP is one of the key problems in logistics that involves servicing many users with vehicles from a depot. The solution that can be obtained by using different algorithms is the path that vehicles should follow when they leave the depot. Therefore, it is necessary to define routes (that incurs minimal costs) so that vehicles leave the depot, serve the maximum number of locations, taking care not to exceed the set limits, and then return to the depot afterward (Ružić et al., 2019). Restrictions to be taken into account are most often related to the capacity of the vehicle, the driver's working time, fleet size and structure etc. The following section briefly explains the algorithms used in the proposed heuristic VRP model.

The Nearest Neighbour (NN) algorithm was used, which is one of the most commonly used algorithms in practice because of its 
simplicity (Toth \& Vigo, 2014). It functions in such a way that when the vehicle leaves the depot, it first serves the nearest location. The next serving location is the one closest to the previously served location, and then this process is repeated until the last location is served. Once all the locations have been serviced, the vehicle returns to the depot, thus ending the route. However, using this algorithm can very often result in solutions that are not close to optimal. For this reason, this algorithm is most commonly used in practice in conjunction with some of the many route enhancement algorithms. The second algorithm used to form the VRP model relates to 2-OPT enhancements. It is used for a reason mentioned above, to improve the route obtained in this case using the $\mathrm{NN}$ algorithm. It works by separating two arcs from the existing route and connecting them differently, taking care not to get two cycles from one route. When all the arcs are connected, the vehicle's total distance is measured and then is being compared with the condition before changing the positions of the arcs. If the savings are achieved, the arcs remain connected; otherwise, they return to their starting position. The procedure is repeated for all possible pairs of arcs in the route. In addition to the two algorithms above, three other enhancement algorithms were used to form the heuristic model, namely Swap, Relocate, and Path Relocate. Unlike the 2-OPT algorithm, which reduces vehicle distances solely within one route, these three algorithms aim to reduce the total distance travelled by two or more vehicles serving locations on the same day or for a different period (Toth \& Vigo, 2014).

A combination of the previous algorithms has formed a heuristic VRP model. First of all, the "giant" route was obtained using the $\mathrm{NN}$, and then it was enhanced with the 2-OPT. Such a route does not meet the vehicle's capacity constraints and the driver's working time, so it needs to be divided into several smaller routes to satisfy it. This is done in a way that the vehicle follows the resulting gigantic route as long as the restrictions allow location maintenance. The first location that would exceed any of the restrictions indicates that the vehicle is returning to the depot, and it becomes the first location to be served by the next route. This produces a greater number of "truncated" routes that satisfy the constraints. These routes then go through the 2-OPT enhancement again and then through the Relocate, Swap, and Path Relocate algorithm. It should be emphasized that the 2-OPT algorithm is repeated between each of the three algorithms mentioned, and the following figure (Figure 1) shows graphically the order of application of the algorithms that make up the VRP model. In this way, the final routes are formed, which represent the operation of the distribution system.

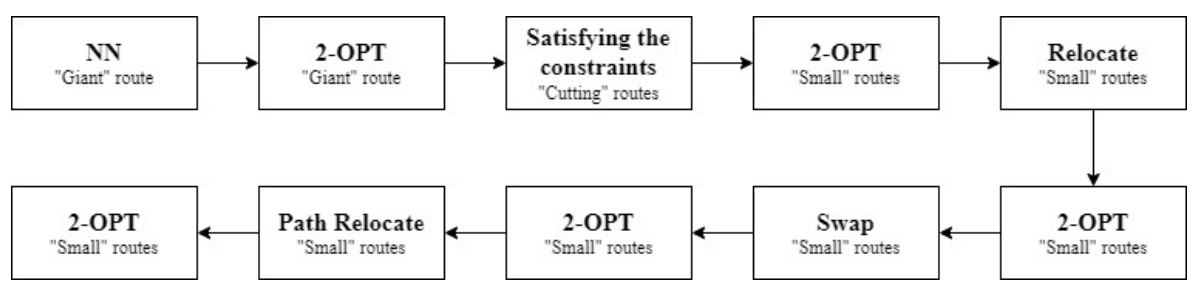

Fig. 1.

The Order of the Applied Algorithms when Forming a VRP Model 


\subsection{Simulation Model}

In addition to the VRP model itself, which will represent the current state, it is also necessary to define the simulation concept used in our approach. It refers to identifying new deliveries that simulate the requirements of a new user entering the system. It is required to determine the parameters based on which they will be formed and then add them to the existing state.

The simulation model itself depends on five input parameters. First of all, it is the number of objects that the user requires for service. This involves randomly selecting a certain number of objects from the set of all objects that were once served in the period under review. When these facilities are chosen, the question arises as to what day their service will be realized. This is defined randomly with a certain probability $P$. It represents the possibility of a specified object being served on a given day. It is this probability that represents the second input parameter of the simulation. After defining it, it is clear on what days the objects will be served, after which it is still necessary to define the number of goods required by the new user. It is expressed in the number of pallets and kilograms per delivery and is determined using three new input parameters. First of all, based on existing data, it is established that the number of pallets appearing in delivery is closest to the exponential distribution. On this basis, the number of pallets of the new user is determined precisely according to the exponential distribution with parameter $\lambda$, which also represents the third input parameter. The last two parameters relate to assessing the weight of delivery that is associated with the number of pallets. As it is determined from the input data that the weight of one pallet can most closely be defined according to the normal distribution, the last two parameters are $\mu$ and $\sigma$ that determine these values.

After identifying the mentioned parameters, simulated deliveries are added to existing ones. For all deliveries, the redefined heuristic VRP model is now being applied. However, since a random event plays a rather significant role in the simulation, the process is repeated. After a large number of iterations, the average value represents the system's final results after the arrival of a new client, and the impact of its appearance can be seen through a comparative analysis of the system before and after arrival.

\section{Computational Results}

To fully understand the effects of applying a defined model, it was tested on a real case example of one 3PL (Third-Party Logistics) company in Serbia. This part of the paper first presents the current case and the input data of the last mile delivery in the observed company. The application of the model and the results of the system before and after the arrival of the new client are presented. Based on these results, the last section presents a comparative analysis of the impact of the arrival of a new user on the system, based on which the price that the provider can offer to the new user can be defined.

\subsection{Review of the Current Case of Last- mile Delivery in the Observed Company}

The solution for the current case of distribution in the logistics company was obtained by applying the VRP model to input data containing information on just over 10000 deliveries that were made in the observed month. Table 2 shows the results obtained by applying the model. The results 
are presented separately for those deliveries that are realized directly and for multi-stop deliveries. In the observed period, according to the obtained data, a distribution was made with a total of 337 routes with a total distance of $41618 \mathrm{~km}$. The total quantity of goods distributed is $347 \mathrm{t}$, which is a total of 1128 pallets. The total cost of distribution for the observed month is 9725 monetary units (m.u.).

Table 2

Current Overview of Last-mile Delivery in the Observed Company

\begin{tabular}{|l|c|c|c|c|c|c|c|c|}
\hline \multirow{2}{*}{ Delivery } & Costs & Distance & Num. of & Num. of & Weight & Num. of & \multicolumn{2}{|c|}{ Monetary Units (m.u.) } \\
\cline { 6 - 10 } & (m.u.) & {$[\mathbf{k m}]$} & Routes & Pll & {$[\mathbf{k g}]$} & Objects & pallet & kg \\
\hline Direct & 510 & 1720 & 27 & 261 & 119021 & 27 & 1.9538 & 0.0043 \\
\hline Multi Stop & 9215 & 39899 & 310 & 867 & 228732 & 3402 & 10.6273 & 0.0403 \\
\hline$\Sigma$ & 9725 & 41618 & 337 & 1128 & 347753 & 3429 & 8.6200 & 0.0280 \\
\hline
\end{tabular}

\subsection{Last-Mile Delivery after a new User arrives in the System}

As mentioned above, the representation of the company after the arrival of a new user (client) can be realized using the simulation model mentioned above. Changing the input parameters explained in detail in the previous chapter can form different types of users and their service requirements. The following is an example of input parameters that can generally display different kinds of users.

First of all, it is necessary to define the number of objects whose service a new user requires. The idea is to use three types of users in the simulation which have different service requirements. Precisely, new users can have a small, medium, or large service requirement with 100,200, and 300 objects respectively in different simulation variants. The second parameter related to probability $\mathrm{P}$ has the idea of representing customers who require higher or lower delivery frequency, so its values are $0.25,0.5$, and 0.75 in different simulation variants. The third input parameter refers to the number of pallets per delivery that is defined by the exponential distribution and can be determined based on the average number of pallets in previous deliveries. Given that the average number of pallets per delivery is 0.4 , the parameter $\lambda$ takes a value of $1 / 0.4$, which is equal to 2.5 . In addition to this expected $\lambda$ value, additionally we observed $\lambda$ reduced by $50 \%$ $(\lambda=1.667)$ as well as increased by $100 \%(\lambda=$ $5)$. The remaining two parameters related to the weight of the pallet (per delivery) are the input parameters of the normal distribution based on which this number is defined. First of all, the mean $\mu$ that represents the average weight of one pallet should be determined. According to the data from the previous period, it amounts to $521.2 \mathrm{~kg}$, and in addition to this value, the values of 260.6 and 781.8 were taken as additional variants in the simulation in order to analyse the sensitivity of solutions. The last parameter $\sigma$ refers to the deviation when defining the weight of the pallet, and it is always 0.1 from $\mu$ (which means that the deviation will have a value of $10 \%$ of the parameter $\mu$ ). The overview of the parameters and values used for defining different simulation variants are presented in Table 3. 
Table 3

The Values of Input Parameters for 81 Different Variants of the Simulation

\begin{tabular}{|c|c|c|c|}
\hline $\begin{array}{c}\text { Number of } \\
\text { Objects }\end{array}$ & Probability $\mathbf{P}$ & $\mathbf{1} / \boldsymbol{\lambda}$ & $\boldsymbol{\mu}$ \\
\hline 100 & 0.25 & 0.2 & 260.6 \\
\hline 200 & 0.50 & 0.4 & 521.1 \\
\hline 300 & 0.75 & 0.6 & 781.8 \\
\hline
\end{tabular}

Since four variable input parameters are defined, which have three different values; it is possible to form 81 different types of a new user (simulation variants). One simulation of 100 iterations was performed for each user, which represents a simulated operation of the distribution system for 100 months. Average iteration values represent the result of each simulation. Table 4 shows the results of one of the simulations related to the arrival of the average user in the system. It requires 200 delivery facilities to be served with a probability of 0.5 . The number of pallets in delivery is defined by an exponential distribution with an expected value of 0.4. In contrast, the weight of each pallet is defined by a normal distribution with an expectation of $521.1 \mathrm{~kg}$ and a deviation of $10 \%$.

Table 4

Overview of Last-mile Delivery in the Observed Company after the Arrival of the New Client

\begin{tabular}{|l|c|c|c|c|c|c|c|c|}
\hline Delivery & \multirow{2}{*}{$\begin{array}{c}\text { Costs } \\
\text { (m.u.) }\end{array}$} & $\begin{array}{c}\text { Distance } \\
{[\mathbf{k m}]}\end{array}$ & $\begin{array}{c}\text { Num. of } \\
\text { Routes }\end{array}$ & $\begin{array}{c}\text { Num. of } \\
\text { Pll }\end{array}$ & $\begin{array}{c}\text { Weight } \\
(\mathbf{k g})\end{array}$ & $\begin{array}{c}\text { Num. of } \\
\text { Objects }\end{array}$ & \multicolumn{2}{|c|}{ Monetary Units (m.u.) } \\
\cline { 5 - 9 } & & & & & & & \\
\hline Direct & 796 & 2719 & 42 & 426 & 186057 & 42 & 1.8690 & 0.0043 \\
\hline Multi Stop & 15464 & 64106 & 505 & 1922 & 774029 & 5194 & 8.0472 & 0.0200 \\
\hline$\Sigma$ & 16261 & 66825 & 548 & 2348 & 960086 & 5237 & 6.9260 & 0.0169 \\
\hline
\end{tabular}

The company's distribution after the arrival of the average user has changed significantly compared to the current one. Total costs increase by as much as $67 \%$ to 16261 m.u. A similar increase occurs with other parameters, so the total distance travelled is $66825 \mathrm{~km}$, the total number of pallets is 2348 , and the total weight is $960 \mathrm{t}$. It is quite clear that as the volume of work increases, the total number of vehicle miles travelled increases, and thus the total cost. However, Table 4 shows unit costs per pallet, as well as unit costs per kilogram of goods distributed. In this case, unit costs are smaller after the arrival of a new client, but they are greatly influenced by the structure of the new customer, that is, his distribution requirements. For this reason, in the next part of the paper, a comparative analysis is made in which the influence of the new user on the existing distribution system, in relation to its characteristics, is seen.

\subsection{Comparative Analysis of Distribution before and after the Arrival of a New Client}

The impact of the arrival of a new customer in the distribution system depends on its characteristics, i.e., the demand for the delivery of goods. Certainly, the user who requires delivery for a small number of 
objects with a low frequency and quantity of delivery will not have the same impact and the user who requires the daily delivery of a large number of objects with large quantities of goods. It is precisely the examination of this influence about the type of users that is the aim of the comparative analysis. First of all, Table 5 shows the unit cost per kilogram concerning all input parameters that determine the characteristics of new users' requests. The unit costs in the table are expressed in m.u. $/ \mathrm{kg}$. The parameter $\mu$ defines the weight of one pallet while the parameter $1 / \lambda$ represents the expected number of pallets. The P mark represents the probability of servicing each user on a given day.

Table 5

Unit Cost per kg after the Arrival of a New Client

\begin{tabular}{|c|c|c|c|c|c|c|c|c|c|c|}
\hline \multirow{2}{*}{\multicolumn{2}{|c|}{ Parameters }} & \multicolumn{9}{|c|}{ Number of Objects } \\
\hline & & \multicolumn{3}{|c|}{100} & \multicolumn{3}{|c|}{200} & \multicolumn{3}{|c|}{300} \\
\hline$\mu$ & $1 / \lambda$ & 0.25 & 0.5 & 0.75 & 0.25 & 0.5 & 0.75 & 0.25 & 0.5 & 0.75 \\
\hline \multirow{3}{*}{260.6} & 0.2 & 0.0302 & 0.0306 & 0.0305 & 0.0307 & 0.0302 & 0.0291 & 0.0305 & 0.0291 & 0.0274 \\
\hline & 0.4 & 0.0279 & 0.0265 & 0.0250 & 0.0264 & 0.0238 & 0.0216 & 0.0250 & 0.0216 & 0.0194 \\
\hline & 0.6 & 0.0260 & 0.0234 & 0.0214 & 0.0234 & 0.0199 & 0.0179 & 0.0214 & 0.0179 & 0.0161 \\
\hline \multirow{3}{*}{521.2} & 0.2 & 0.0277 & 0.0262 & 0.0247 & 0.0261 & 0.0233 & 0.0211 & 0.0247 & 0.0211 & 0.0187 \\
\hline & 0.4 & 0.0239 & 0.0207 & 0.0186 & 0.0207 & 0.0169 & 0.0150 & 0.0185 & 0.0150 & 0.0134 \\
\hline & 0.6 & 0.0212 & 0.0177 & 0.0156 & 0.0176 & 0.0143 & 0.0129 & 0.0156 & 0.0129 & 0.0118 \\
\hline \multirow{3}{*}{781.8} & 0.2 & 0.0255 & 0.0228 & 0.0209 & 0.0229 & 0.0193 & 0.0170 & 0.0209 & 0.0170 & 0.0151 \\
\hline & 0.4 & 0.0212 & 0.0176 & 0.0156 & 0.0176 & 0.0144 & 0.0129 & 0.0156 & 0.0129 & 0.0118 \\
\hline & 0.6 & 0.0187 & 0.0152 & 0.0136 & 0.0153 & 0.0127 & 0.0116 & 0.0136 & 0.0116 & 0.0107 \\
\hline
\end{tabular}

Table 5 shows that the unit costs vary and, in some cases, are higher and, in some cases, lower than the unit costs of the current distribution, which are $0.0280 \mathrm{~m} . \mathrm{u} . / \mathrm{kg}$. For this reason, it is necessary to present the results so that one can see in which cases and in what quantity unit costs are increasing or decreasing. Table 6 shows the cost savings per unit cost per kilo by the arrival of a new customer who has certain characteristics of a delivery request. All values are expressed in percentages; negative ones are those where losses occur, i.e., unit costs increase, while positive values indicate savings and unit costs decrease.

\section{Table 6}

Savings per Unit Cost per Pound upon Arrival of a New Client

\begin{tabular}{|c|c|c|c|c|c|c|c|c|c|c|}
\hline \multirow{2}{*}{\multicolumn{2}{|c|}{ Parameters }} & \multicolumn{9}{|c|}{ Number of Objects } \\
\hline & & \multicolumn{3}{|c|}{100} & \multicolumn{3}{|c|}{200} & \multicolumn{3}{|c|}{300} \\
\hline$\mu$ & $1 / \lambda$ & 0.25 & 0.5 & 0.75 & 0.25 & 0.5 & 0.75 & 0.25 & 0.5 & 0.75 \\
\hline \multirow{3}{*}{260.6} & 0.2 & $-8 \%$ & $-10 \%$ & $-9 \%$ & $-10 \%$ & $-8 \%$ & $-4 \%$ & $-9 \%$ & $-4 \%$ & $2 \%$ \\
\hline & 0.4 & $0 \%$ & $5 \%$ & $10 \%$ & $5 \%$ & $15 \%$ & $23 \%$ & $11 \%$ & $23 \%$ & $31 \%$ \\
\hline & 0.6 & $7 \%$ & $16 \%$ & $23 \%$ & $16 \%$ & $29 \%$ & $36 \%$ & $23 \%$ & $36 \%$ & $42 \%$ \\
\hline \multirow{3}{*}{521.2} & 0.2 & $1 \%$ & $6 \%$ & $12 \%$ & $7 \%$ & $17 \%$ & $25 \%$ & $12 \%$ & $25 \%$ & $33 \%$ \\
\hline & 0.4 & $14 \%$ & $26 \%$ & $34 \%$ & $26 \%$ & $39 \%$ & $46 \%$ & $34 \%$ & $46 \%$ & $52 \%$ \\
\hline & 0.6 & $24 \%$ & $37 \%$ & $44 \%$ & $37 \%$ & $49 \%$ & $54 \%$ & $44 \%$ & $54 \%$ & $58 \%$ \\
\hline \multirow{3}{*}{781.8} & 0.2 & $9 \%$ & $18 \%$ & $25 \%$ & $18 \%$ & $31 \%$ & $39 \%$ & $25 \%$ & $39 \%$ & $46 \%$ \\
\hline & 0.4 & $24 \%$ & $37 \%$ & $44 \%$ & $37 \%$ & $49 \%$ & $54 \%$ & $44 \%$ & $54 \%$ & $58 \%$ \\
\hline & 0.6 & $33 \%$ & $46 \%$ & $51 \%$ & $45 \%$ & $55 \%$ & $59 \%$ & $51 \%$ & $59 \%$ & $62 \%$ \\
\hline
\end{tabular}


Table 6 shows two extreme cases. The first refers to the arrival of the customer, which requires extremely small deliveries, in which case unit costs per kilogram can be increased, which is a loss for the company. This is because new delivery locations are emerging that are not currently being served, and there are not enough goods to justify their visit. In other words, it is necessary to include new locations in the route, whose visit requires additional costs, while delivering a small number of goods, which is certainly not profitable for distribution. On the other hand, when a user enters the system, there is a situation that requires an extremely large amount of goods compared to the existing system. In this case, unit costs can be reduced by over $50 \%$. This is because the new user requires a large number of service locations; the network becomes denser and therefore more convenient for the distribution of goods. Also, a large number of locations coincide with those currently being served, causing some of them to exceed the defined limit (2.5 $t$ and 8 pll) and become direct deliveries that are realized by larger vehicles.

However, when the mentioned extreme cases are omitted and when the average values of input parameters that represent the requirements of average users are observed, it can be said that the arrival of a new user in the mentioned distribution system can save $5-35 \%$ in unit costs per kilogram. Table 6 shows that each of the input parameters has a significant impact on savings. For example, a new customer who requires delivery of goods in 100 different facilities with a facility service probability per day (parameter $\mathrm{P}$ ) of 0.5 , and an expected number of pallets in delivery (parameter $1 / \lambda$ ) of 0.4 , has very different savings values depending on the weight of one pallet in delivery (parameter $\mu$ ). If the expected weight of the pallet according to the normal distribution is $260.6 \mathrm{~kg}$, savings in unit cost per kilogram of $5 \%$ are achieved. When this weight is equal to $521 \mathrm{~kg}$, the savings are significantly higher and amount to $26 \%$, while in the case of a pallet weight of $781.8 \mathrm{~kg}$; the savings can be up to $37 \%$. This means that each of the characteristics of a potential customer has a significant impact on the price of the service offered to him. It must be defined separately for each customer.

In addition to unit costs per kilogram, another criterion was observed - unit cost per number of pallets. The following table (Table 7) shows the unit cost per pallet after the arrival of a potential client.

\section{Table 7}

Savings per Unit Cost per Pallet upon Arrival of a New Client

\begin{tabular}{|c|c|c|c|c|c|c|c|c|c|c|}
\hline \multirow{2}{*}{\multicolumn{2}{|c|}{ Parameters }} & \multicolumn{9}{|c|}{ Number of Objects } \\
\hline & & \multicolumn{3}{|c|}{100} & \multicolumn{3}{|c|}{200} & \multicolumn{3}{|c|}{300} \\
\hline$\mu$ & $1 / \lambda$ & 0.25 & 0.5 & 0.75 & 0.25 & 0.5 & 0.75 & 0.25 & 0.5 & 0.75 \\
\hline \multirow{3}{*}{260.6} & 0.2 & 9.100 & 9.082 & 8.935 & 9.111 & 8.749 & 8.254 & 8.932 & 8.270 & 7.642 \\
\hline & 0.4 & 8.277 & 7.665 & 7.124 & 7.651 & 6.662 & 5.945 & 7.103 & 5.938 & 5.274 \\
\hline & 0.6 & 7.599 & 6.666 & 5.975 & 6.673 & 5.509 & 4.859 & 5.983 & 4.872 & 4.338 \\
\hline \multirow{3}{*}{521.2} & 0.2 & 9.128 & 9.127 & 8.988 & 9.118 & 8.776 & 8.360 & 8.988 & 8.342 & 7.777 \\
\hline & 0.4 & 8.339 & 7.796 & 7.326 & 7.794 & 6.926 & 6.422 & 7.297 & 6.411 & 5.992 \\
\hline & 0.6 & 7.673 & 6.944 & 6.433 & 6.926 & 6.116 & 5.740 & 6.435 & 5.734 & 5.443 \\
\hline \multirow{3}{*}{781.8} & 0.2 & 9.153 & 9.146 & 9.040 & 9.159 & 8.884 & 8.571 & 9.057 & 8.564 & 8.228 \\
\hline & 0.4 & 8.436 & 8.029 & 7.764 & 8.033 & 7.560 & 7.349 & 7.739 & 7.344 & 7.192 \\
\hline & 0.6 & 7.959 & 7.513 & 7.264 & 7.521 & 7.140 & 7.019 & 7.279 & 7.011 & 6.927 \\
\hline
\end{tabular}


As previously shown, the current unit cost per pallet is $8.62 \mathrm{~m} . \mathrm{u} . / \mathrm{pll}$. After the arrival of a potential client in the system, this cost varies from 4.338 m.u./pll to 9.159 m.u./pll. In order to present this relationship more clearly, the following table shows the percentage savings of unit costs per pallet, which is achieved by the arrival of a potential user in the system.

Table 8

Percentage Savings per Unit Cost per Pallet upon arrival of a New Client

\begin{tabular}{|c|c|c|c|c|c|c|c|c|c|c|}
\hline \multirow{2}{*}{\multicolumn{2}{|c|}{ Parameters }} & \multicolumn{9}{|c|}{ Number of Objects } \\
\hline & & \multicolumn{3}{|c|}{100} & \multicolumn{3}{|c|}{200} & \multicolumn{3}{|c|}{300} \\
\hline$\mu$ & $1 / \lambda$ & 0.25 & 0.5 & 0.75 & 0.25 & 0.5 & 0.75 & 0.25 & 0.5 & 0.75 \\
\hline \multirow{3}{*}{260.6} & 0.2 & $-6 \%$ & $-5 \%$ & $-4 \%$ & $-6 \%$ & $-2 \%$ & $4 \%$ & $-4 \%$ & $4 \%$ & $11 \%$ \\
\hline & 0.4 & $4 \%$ & $11 \%$ & $17 \%$ & $11 \%$ & $23 \%$ & $31 \%$ & $18 \%$ & $31 \%$ & $39 \%$ \\
\hline & 0.6 & $12 \%$ & $23 \%$ & $31 \%$ & $23 \%$ & $36 \%$ & $44 \%$ & $31 \%$ & $43 \%$ & $50 \%$ \\
\hline \multirow{3}{*}{521.2} & 0.2 & $-6 \%$ & $-6 \%$ & $-4 \%$ & $-6 \%$ & $-2 \%$ & $3 \%$ & $-4 \%$ & $3 \%$ & $10 \%$ \\
\hline & 0.4 & $3 \%$ & $10 \%$ & $15 \%$ & $10 \%$ & $20 \%$ & $25 \%$ & $15 \%$ & $26 \%$ & $30 \%$ \\
\hline & 0.6 & $11 \%$ & $19 \%$ & $25 \%$ & $20 \%$ & $29 \%$ & $33 \%$ & $25 \%$ & $33 \%$ & $37 \%$ \\
\hline \multirow{3}{*}{781.8} & 0.2 & $-6 \%$ & $-6 \%$ & $-5 \%$ & $-6 \%$ & $-3 \%$ & $1 \%$ & $-5 \%$ & $1 \%$ & $5 \%$ \\
\hline & 0.4 & $2 \%$ & $7 \%$ & $10 \%$ & $7 \%$ & $12 \%$ & $15 \%$ & $10 \%$ & $15 \%$ & $17 \%$ \\
\hline & 0.6 & $8 \%$ & $13 \%$ & $16 \%$ & $13 \%$ & $17 \%$ & $19 \%$ & $16 \%$ & $19 \%$ & $20 \%$ \\
\hline
\end{tabular}

As expected, the most considerable impact on unit costs per pallet has the parameter $1 / \lambda$, which refers to the number of pallets that potential users require per delivery. Increasing the number of pallets per delivery increases the number of goods distributed, which affects the reduction of unit cost per pallet. However, the parameter $\mu$, which refers to one pallet's weight, also significantly impacts savings. The heavier the pallet, the lower the unit cost savings per pallet. This is a consequence of "faster" reaching the weight limit when forming routes. So, if we look at a certain number of pallets that need to be distributed, and there is an option that the pallets are heavier or lighter, it is logical that when defining the route in heavier pallets, some of the vehicle limitations are reached. If the pallet's weight is lower, and the number of pallets remains the same, the vehicle's weight limit has a smaller role in defining the routes, which leads to higher utilization of the vehicle per pallet, thus to lower unit costs.

As with unit costs per kilogram, extreme cases can be seen here where there are considerable savings in unit costs of $50 \%$. Theoretically, this case is possible, but in practice, one should consider the capacity of the fleet of vehicles used for routing, which is certainly limited. The arrival of an extensive client in the existing distribution system significantly reduces unit costs. Still, we should not forget that this entails large investments in the vehicle fleet and other resources necessary for distribution to be realized.

This model's advantage in practice is that when defining the offer, the parameters are defined only for one user to whom the service is offered. This enables a much faster solution, as well as an increase in the number of iterations, which allows more precise output results.

The following figures (Figure 2 and Figure 3) show an example of routes that are realized one day. On the first above (Figure 2) are routes representing the current distribution status, while on the second after (Figure 3) are routes representing a new user's arrival. According to the input data, the black 
locations are certainly served on an observed day, while the black with white dot ones are the deliveries that are simulated or required by the new user. The picture shows that some of the new locations are already being served at the current distribution of goods (black grey with white dot), while some of them are brand new in the system (grey with white dot). It should also be mentioned that the grey branches represent direct deliveries and that the square represents the depot or distribution centre.

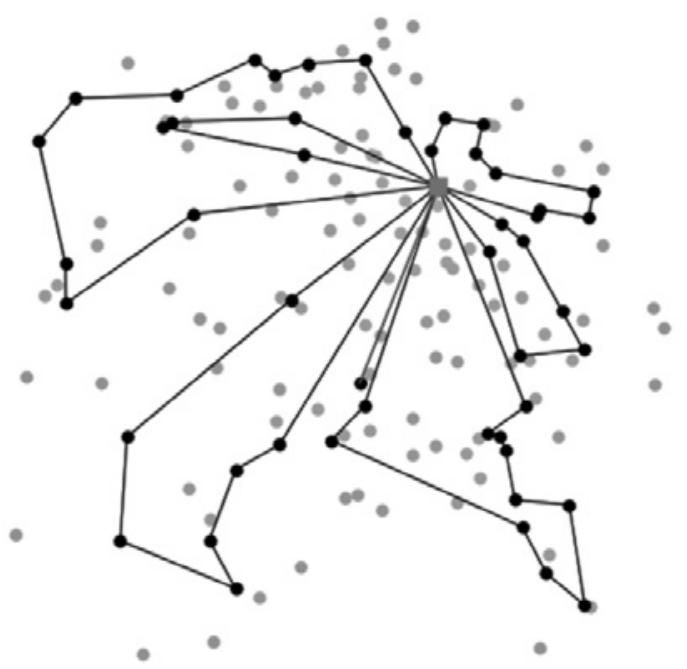

Fig. 2.

Example of Routes before New User Arrival

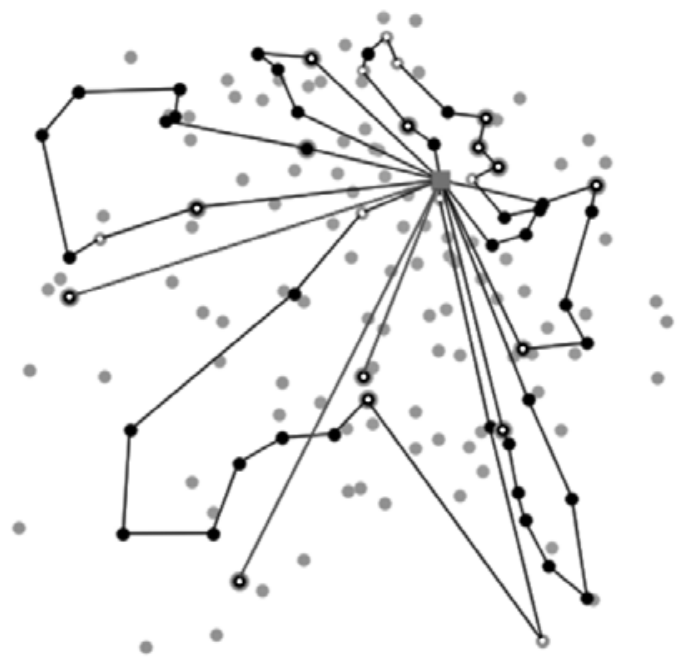

Fig. 3.

Example of Routes after New User Arrival 
Thus, with the increase in the number of goods, the number of direct deliveries increases, since the locations reach the limit of $2.5 \mathrm{t}$ and eight pallets. However, when observing the share of direct and multistop deliveries, the situation is somewhat different. In the specific example given (Figure 4), the average user was observed. "Average potential user" means that the expected number of pallets required by the user is 0.4 with an expected pallet weight of $521.2 \mathrm{~kg}$. Also, the probability $\mathrm{P}$ is 0.5 , and the number of required deliveries in the observed period is 200 .

Of course, the total weight of goods directly distributed to the user is significantly increased in this case, from $119 \mathrm{t}$ to $186 \mathrm{t}$. The situation is similar to the number of pallets, which in the current state is 261 pallets, while after the arrival of the average potential user in the system, this number increases to 426 pallets. However, it should be noted that the potential user's expected input parameters are significantly lower than the limit of $2.5 \mathrm{t}$ and eight pallets. This means that the potential user can influence the delivery to become direct, but only if there is already a certain amount of goods being delivered at the observed location. On the other hand, due to the smaller quantity of goods, most simulated orders involve routing, which significantly affects multistop delivery. Figure 4 shows that $34 \%$ of the weight of goods in the current distribution state is delivered directly from the regional center to the user. The remaining $66 \%$ of the total weight of the goods is delivered by routing, where more than one user is served simultaneously. After simulating the average user's entry into the system, the ratio of direct and multi-stop deliveries changes. In this case, $19 \%$ of the goods' weight is delivered directly, while the other $81 \%$ is delivered by routing. When it comes to the number of pallets, $23 \%$ of pallets are delivered directly before the user arrival simulation, while the rest of the pallets are delivered through multi-stop deliveries. After the user enters the system, this percentage is reduced to $18 \%$, while the remaining $82 \%$ of pallets are delivered through vehicle routing.

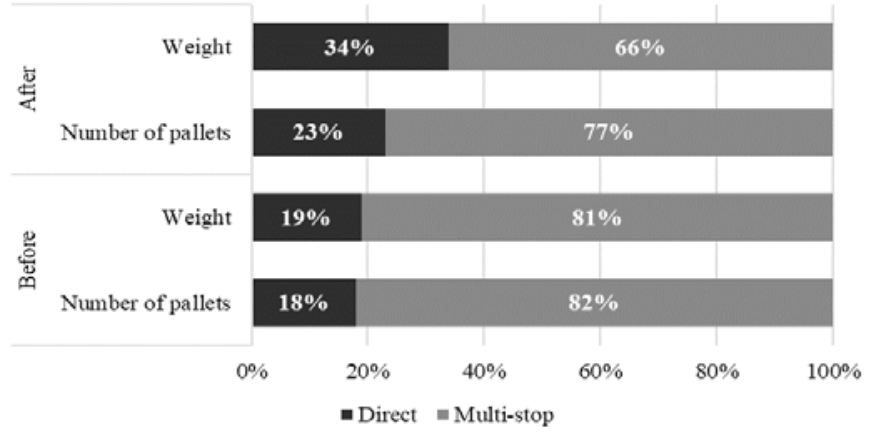

Fig. 4.

An Example of the Relationship between Direct and Multi-stop Deliveries before and after the Simulated Arrival of Users in the System 


\section{Conclusion}

This work's contribution is reflected in the development of a simulation-optimization model that aims to look at the impact of the arrival of a new user in the existing distribution system. First of all, using different heuristic algorithms, the VRP model was formed. The second part of the paper deals with its application on real data provided by a logistics company in Serbia. This is where the current distribution state is obtained, and then the state after the new user arrives in the system. At the end of the paper, a comparative analysis was made, where the impact of the arrival of a new user was considered.

Based on the output, it is possible to define an adequate price for the service that can be offered to a particular user, depending on his requirements. In determining the price, the provider must be extremely careful because, as shown, some users can lead to an increase in unit distribution costs. However, most of the analyzed types of users have a positive impact on the distribution system. Of course, increasing the volume of work requires a more complex process of realizing the distribution of goods. Still, unit costs can be significantly reduced by the arrival of new users. In defining the price, the logistics company must cover all distribution costs and, at the same time, offer a low enough price to the user to compete with the market. Of course, this model may help in deciding on the offer, but in the future, it may come closer to the real world. One of the future improvements of the presented approach can be the introduction of time windows, where each user will have a defined time when he can receive the goods or application of other VRP heuristics and improvement algorithms.

\section{Acknowledgments}

This work was supported by the Ministry of Education, Science, and Technical Development of the Government of the Republic of Serbia [grant number TR36006], for the period 2011-2020.

\section{References}

Gevaers, R.; Van de Voorde, E.; Vanelslander, T. 2014. Cost Modelling and Simulation of Last-mile Characteristics in an Innovative B2C Supply Chain Environment with Implications on Urban Areas and Cities, Procedia - Social and Behavioral Sciences 125: 398411 .

Drenovac, D.; Vidović, M.; Bjelić N. 2020. Optimization and simulation approach to optimal scheduling of deteriorating goods collection vehicles respecting stochastic service and transport times, Simulation Modelling Practice and Theory 103: 102097. doi: https:// doi.org/10.1016/j.simpat.2020.102097.

Mičeta, D.; Ostojić, I.; Marković, L.; Stojanović, F. 2019. Periodic replenishment with zoning: A distribution company case study. In Proceedings of the 4th Logistic International Conference - Logic 2019, Belgrade, Serbia, 328-334.

Ružić, M.; Popović, D.; Mičeta, D. 2019. A heuristic approach to solving periodic vehicle routing problem with zoning. In Proceedings of the XLVI International Symposium on Operational Research - Symopis 2019, Kladovo, Serbia, 315-320.

Teodorović, D. 2016. Transport Networks [In Serbian: Transportne mreže]. University in Belgrade, Faculty of Transport and Traffic, Serbia. 429 p.

Toth, P.; Vigo, D. 2014. Vehicle Routing: Problems, Methods and Applications. Second Edition, Society for Industrial and Applied Mathematics, Philadelphia, USA. 